\title{
Prevención de Factores de Riesgo en Adolescentes: Intervención para Padres y Madres
}

\author{
Silvia Guadalupe Argaez Rodríguez';https://orcid.org/0000-0002-2866-0786 \\ Rebelín Echeverría Echeverría1; https://orcid.org/0000-0002-1331-2367 \\ Nancy Marine Evia Alamilla1; https://orcid.org/0000-0002-5652-7325 \\ Carlos David Carrillo Trujillo1; https://orcid.org/0000-0003-0228-9293
}

\begin{abstract}
Resumen
Los adolescentes experimentan diversos cambios, los cuales, en ocasiones, pueden ponerlos en riesgo. El objetivo fue conocer los factores de riesgo a los que están expuestos adolescentes de secundaria en Mérida, Yucatán, para posteriormente analizarlos y proponer acciones de prevención en una intervención dirigida a sus padres y madres. La metodología se basó en la investigación acción participativa, en tres fases. En la primera, el diagnóstico social, participaron 191 alumnos de segundo grado y 2 profesoras. En la segunda y la tercera, intervención y evaluación, participaron 30 madres y 6 padres. Las técnicas fueron encuesta, entrevista, grupo de discusión y técnicas participativas. Los principales factores de riesgo identificados son el bullying, la violencia familiar y el alcoholismo. Los padres y madres manifiestan un genuino interés por formarse y tomar medidas preventivas. Se discute la importancia del contexto social y familiar para favorecer la prevención de dichos factores en la adolescencia.
\end{abstract}

Keywords: Factores de riesgo; adolescente; prevención.

\section{Prevention of Risk Factors in Adolescents: Intervention for Parents and Mothers}

\begin{abstract}
Adolescents experience various changes, which can sometimes put them at risk. The objective was to know the risk factors to which high school adolescents are exposed in Mérida, Yucatán, to later analyze those risks and propose prevention actions in an intervention addressed to their parents. The methodology was based on participatory action research, in three phases. In the first, the social diagnosis, 191 second grade students and 2 female teachers participated. In the second and third, intervention and evaluation, 30 mothers and 6 fathers participated. The techniques were survey, interview, discussion group and participatory techniques. The main risk factors identified are bullying, family violence and alcoholism. Parents express a genuine interest in informing themselves and taking preventive measures. The importance of the social and family context to favor or limit these risk factors in adolescence is discussed.
\end{abstract}

Key words: Risk Factors; adolescent; prevention.

\section{Prevenção de Fatores de Risco em Adolescentes: Intervenção para Pais e Mães}

\section{Resumo}

Os adolescentes experimentam diversas mudanças, as quais, em ocasiões, podem colocá-los em risco. O objetivo foi conhecer os fatores de risco aos que estão expostos - adolescentes do ensino médio em Mérida, Yucatán, para posteriormente analisá-los e propor ações de prevenção em uma intervenção dirigida a seus pais e mães. A metodologia se baseou na investigação ação participativa, em três fases. Na primeira, o diagnóstico social, participaram 191 alunos do ensino médio e duas professoras. Na segunda e na terceira, intervenção e avaliação, participaram 30 mães e seis pais. As técnicas foram questionário, entrevista, grupo de discussão e técnicas participativas. Os principais fatores de risco identificados são o bullying, a violência familiar e o alcoolismo. Os pais e mães manifestaram um genuíno interesse por informar-se e tomar medidas preventivas. Discute-se a importância do contexto social e familiar para favorecer a prevenção dos ditos fatores na adolescência.

Palavras-chave: Fatores de risco; adolescente; prevenção.

1 Facultad de Psicología, Universidad Autónoma de Yucatán - Yucatán - México; friend_30_91@hotmail.com; rebelin.echeverria@gmail.com; nancy.evia@gmail.com; carrillo.trujillo@gmail.com 


\section{Introducción}

\section{Factores del riesgo en la adolescencia}

La adolescencia representa un periodo crítico en el inicio y afianzamiento de conductas de riesgo (Tarín \& Navarro, 2006), las cuales se presentan como la evidencia práctica y concreta de la exposición a factores de riesgo. De acuerdo con la Organización Mundial de la Salud (2013) un factor de riesgo es, cualquier rasgo, característica o exposición de un individuo a que incremente su probabilidad de sufrir una enfermedad o lesión. Los factores de riesgo implican determinadas conductas de riesgo, las cuales son acciones activas o pasivas que involucran peligro para el bienestar $y$, que conllevan directamente a consecuencias negativas para la salud o comprometen aspectos del desarrollo. Así, las conductas de riesgo son aquellas acciones voluntarias o involuntarias, realizadas por el individuo o comunidad, que pueden llevar a consecuencias nocivas (Fonseca, 2010 en Rosabal García, Romero Muñoz, Gaquín Ramírez, Hernández Mérida, 2015).Ejemplos de éstas son: las conductas delictivas y la conducta sexual de riesgo(Lila, Buelga, \& Musitu, 2006), el consumo de alcohol, tabaco y otras drogas, deserción escolar y relación sexual precoz, entre otras (Idrobo, Castellano, \& Nieto, 1998, en Vázquez, 2008). De manera particular, este estudio se centra en algunos de los principales factores de riesgo que pueden repercutir de manera negativa en el desarrollo de los adolescentes a saber, los trastornos alimenticios, el suicidio, las autolesiones, el bullying, vida sexual de riesgo, adicción y delincuencia juvenil.

Los Trastornos Alimenticios inician en la edad de los 12 hasta los 25 años y la frecuencia aumenta entre los 12 y los 17 años (Infogen, 2015). Suelen presentarse tanto en hombres como en mujeres, aunque en mujeres es superior, ya que éstas parecen estar más influidas por el ideal de delgadez y expresan insatisfacción con su peso. Este tipo de insatisfacción y la excesiva atención colocada a la imagen corporal, son factores de riesgo para el desarrollo de un trastorno alimentario (Kiefer, Leitner, Bauer, \& Rieder, 2000). En Yucatán, se realizó un estudio con estudiantes, el cual arrojó que el $48 \%$ presentaba una interiorización del modelo estético de delgadez relacionado a la insatisfacción con la figura y peso corporal (Cruz, Ávila, Velázquez, \& Estrella, 2007). Sin embargo, aunque en diferentes discursos y publicaciones periodísticas se expresa una alta presencia de Trastornos de la Alimentación en adolescentes en Yucatán, la investigación científica en dicho campo, aún es casi inexistente. Algunos estudios relacionados son el de Marín Cárdenas, Sánchez Ramírez y Maza Rodríguez (2014) que se centran en la obesidad y hábitos alimenticios de una comunidad del interior del estado y el de Oliva-Peña, Ordóñez-Luna, Santana-Carvajal, Marín-Cárdenas, Andueza Pech y Gómez Castillo (2016) que se centra en analizar la concordancia del Índice de Masa Corporal (IMC) y la percepción de la imagen corporal en adolescentes de una localidad suburbana de Yucatán.
Por otro lado, en México, de acuerdo con Garduño, Gómez y Peña (2011) el intento de suicidio es común entre los adolescentes con predisposición a este tipo de conducta. De manera especial, las tasas de suicidio han ido incrementando entre los adolescentes de 15 años y los jóvenes de 25 años. Es importante destacar que las muertes por suicidio se dan mayormente en hombres, a diferencia de las mujeres en quienes es más común el intento de suicidio (Garduño \& cols., 2011). En México, de acuerdo con el INEGI (2009), a través del documento Estadística de suicidios de los Estados Unidos Mexicanos, desarrollado para difundir información acerca de las muertes autoinfligidas registradas en el ámbito nacional, durante el mismo año, se llevaron a cabo 5190 suicidios, de los cuales, 4201 eran varones y 989 mujeres. Los adolescentes y jóvenes entre 15 y 24 años, ocupan el primer lugar en sectores vulnerables (Subsecretaría de prevención y promoción de la salud, 2011). Las muertes por suicidio en jóvenes se han duplicado en los últimos 30 años. Para el 2013, la prevalencia de intento de suicidio de adolescentes en Yucatán es de 2.6\% (Instituto Nacional de Salud Pública, 2013). Respecto a las edades se presentó un descenso al consumar el acto suicida, hay casos desde los 11 años (Comisión de Derechos Humanos del Estado de Yucatán, 2010). De acuerdo con declaraciones públicas de autoridades sanitarias, el suicidio aumentó durante el 2016 en comparación con el 2015, con mayor número de suicidios en hombres que en mujeres. Pese a la existencia de registros estadísticos que demuestran que el suicidio representa un problema real en los adolescentes, se carece de investigación al respecto.

Por su parte, las autolesiones hacen referencia a la acción de agredirse o dañarse a uno mismo de manera intencional como el pegarse, cortarse o quemarse la piel, entre otras. Las lesiones auto infligidas intencionalmente son la segunda causa de muerte entre los jóvenes, siendo el $20.6 \%$ de las muertes registradas en los varones y el $14 \%$ en las mujeres (INEGI, 2013). Un estudio realizado en la ciudad de México evidenció diversas razones que llevan a los adolescentes de 12 a 17 años a autolesionarse: el $42.1 \%$ se asocia con baja tolerancia a la frustración y manipulación, el $30.6 \%$ a síntomas afectivos como tristeza o irritabilidad o para "lograr sentir algo y sentirse vivos", entre otras. (Ulloa, Hernández, Paniagua, \& Figueroa, 2013).

De acuerdo con Sánchez Ramírez y Dzib Aguilar (2014) quienes realizaron un estudio al respecto con la participación de 1,623 alumnos de primer ingreso de bachillerato, los resultados evidencian que las autolesiones en jóvenes suelen presentarse en cualquier nivel socioeconómico. La forma más común son los cortes en la piel, que no ponen en riesgo la vida de quien lo practica y se relacionan con desórdenes de la alimentación, golpes intencionales y conductas de riesgo en cuanto al consumo de sustancias adictivas. Respecto a esta problemática de autolesiones, se observa una falta de investigaciones tanto a nivel nacional como en el Estado de Yucatán; vacío que hace parecer que ésta no es una problemática actual contra la salud de las personas.

Por su parte, con relación al bullying o acoso escolar, en un estudio realizado por Rocha y Pacheco (2008) 
en 18 secundarias de Mérida con grupos de 15 jóvenes de segundo grado, se encontraron los siguientes resultados: en cuanto a la exclusión, el $74.6 \%$ de los jóvenes señala que en sus escuelas hay compañeros a quienes se les ignora y se le deja solos, mientras que $59 \%$ indicó que hay a quienes los propios jóvenes no les permiten participar en las actividades. El $85.4 \%$ observaba la presencia de apodos ofensivos entre compañeros y el $85.5 \%$ dijo que sus compañeros hablan mal, los unos de los otros. Por otro lado, el $78.2 \%$ de los observadores reportaron ocurrencia de insultos en las escuelas. La identificación de golpes registró una presencia de $66.3 \%$. Respecto a la intimidación, el $56.2 \%$ respondió que esto ocurre en sus escuelas, mientras que la intimidación con palos, navajas $u$ otros objetos fue mucho menos observada con el $12.9 \%$. Las amenazas para obligar a alguien a realizar acciones no deseadas alcanzaron el $45.8 \%$. Finalmente, el acoso sexual se reportó para ambos sexos con una frecuencia de $20.2 \%$.Es importante destacar que, pese a que en el Estado de Yucatán existe una Ley para la Prevención, Combate y Erradicación de la Violencia en el Entorno Escolar (Gobierno del Estado de Yucatán, 2013), el bullying sigue presente y se requiere mayor investigación al respecto.

Con respecto a la sexualidad, de acuerdo con el Consejo Nacional de Población (2014), en Yucatán, la edad promedio de inicio sexual es de 16.1 y el $54.8 \%$ hace uso de métodos anticonceptivos, de los cuales el $86 \%$ usa condón, el $2.9 \%$ usa métodos hormonales y el $11.1 \%$ utiliza otro método; mientras que a nivel nacional la edad de la primera relación es de 15.9 y solo el $37.6 \%$ utiliza algún método anticonceptivo (Consejo Nacional de Población, 2014). A pesar de que actualmente se difunde mucha información al respecto, los adolescentes están en riesgo de contraer Infecciones de Transmisión Sexual (ITS) y/o vivir embarazos a temprana edad.

Por su parte, las adicciones representan una problemática actual que enfrenta la sociedad y los jóvenes en particular. De acuerdo con la Encuesta Nacional de Juventud (2010), en Yucatán, el $16.4 \%$ de los jóvenes entre 12 y 29 años de edad han consumido alguna vez tabaco; el $42.6 \%$ de los jóvenes han consumido alguna bebida alcohólica. Por otro lado, el Instituto Nacional de Salud Pública (2013), señala que el $8.1 \%$ de la población adolescente de 15 a 19 años de edad, ha fumado más de 100 cigarros en su vida, el $14.8 \%$ comenzó a los 12 años o antes y el $85.2 \%$ inició entre los 13 y los 19 años. El Centro de Integración Juvenil (CIJ) reporta que desde el año 2009 se ha incrementado en un $25 \%$ el número de menores de edad a quienes brindan tratamiento por consumo de alcohol (Diario de Yucatán, 2014).

La delincuencia juvenil alcanza, su mayor expresión entre los 13 y 15 años de edad, ya que es un periodo en el cual el menor tiende a relacionarse con otros jóvenes de su edad (Jiménez, 2005). Un aspecto importante es el hecho de que tener un grupo de amistades delincuentes predice problemas escolares y comportamientos antisociales, lo que puede llevar a delinquir (Lodewijk, Doreleijers y De Ruiter, 2008). También resulta importante considerar la influencia que tiene el entorno (Jiménez, 2005). De acuerdo con datos de INEGI en 2010, respecto al fuero común, de 2000 a 2008, los jóvenes de 16 a 19 años constituyeron el 50\% de los presuntos delincuentes del país en materia penal (Banco Mundial, 2012). Existe una carencia de investigaciones a nivel local dedicadas a delincuencia juvenil; sin embargo, en la cotidianeidad se habla de diversas acciones delictivas llevadas a cabo por adolescentes, e incluso, niños.

Finalmente, en México, se reconoce la violencia como un problema de salud pública, ya que influye en la salud de las personas en todas las áreas de su desarrollo. Los grupos de edad más afectada es el de 20-24 años y 25-44 años con importante predominio en el sexo femenino y en el hogar. Respecto al grupo de mujeres de 15 años de edad y más, se encuentra mayor prevalecía de violencia basada en la inequidad de género (Secretaría de Salud de Yucatán, 2015). Sin embargo, la carencia de investigaciones al respecto no permite tener un panorama completo de dicha problemática.

\section{EI papel de la familia ante el riesgo de los adolescentes}

La familia representa una institución que ha permanecido a lo largo de la historia de la humanidad. Según Murueta y Osorio (2009), la familia es considerada como el primer entorno natural en donde los miembros que la forman evolucionan y se desarrollan en términos afectivos, físicos, intelectuales y sociales, según modelos vivenciados e interiorizados. Asimismo, se le ha reconocido como la mayor fuente de apoyo social y personal del que pueden disponer las personas en cualquier momento de sus vidas, tanto en los periodos de independencia como en los de dependencia (niñez, enfermedad). Sin embargo, muchos padres protegen a sus hijos durante los primeros años de la vida, pero a medida que éstos van creciendo, los padres van "cesando» ante dicha protección y más tarde, ante los "nuevos" problemas, recuperar el diálogo perdido resulta una tarea difícil (Redondo Romero, 2013).Se requiere del compromiso de la familia para superar los riesgos y vulnerabilidades propias de esta etapa y estimular adecuadamente su desarrollo (Valenzuela, Ibarra, Zubarew, \& Loreto, 2013).

La importancia de la familia en la prevención de conductas de riesgo se debe a que es un primer agente protector y facilita el desarrollo saludable del adolescente; es en la familia donde se conoce y aprende, la utilidad de dichos aprendizajes puede ayudar a que el adolescente sobrelleve los cambios de manera adecuada (Valenzuela \& cols., 2013). Resulta fundamental que los padres y madres de familia conozcan y comprendan las diferentes etapas del desarrollo humano, para así poder comprender las diferentes conductas de sus adolescentes. Cuando se conocen los elementos que sitúan a un adolescente, en situación de riesgo o si se conoce qué factores pueden disminuir los efectos negativos, los padres pueden poner en marcha estrategias que favorezcan la protección y minimicen dicho riesgo. 
Los resultados de investigaciones llevadas a cabo han demostrado que la falta de comunicación entre padres e hijos incrementa las posibilidades de conductas violentas (Lodewijk \& cols., 2008). Además de que cuando los menores viven expuestos a la violencia familiar, pueden llegar a ver la violencia como la única alternativa a verse convertido en víctima (Secretaría de Educación de Gobierno del Estado y Sindicato Nacional de Trabajadores de la Educación, 2011). Finalmente, es importante destacar que, de acuerdo con Valenzuela, Ibarra, Zubarew y Loreto (2013), existen diversas investigaciones que señalan que cuando los padres y madres de familia son capacitados en el desarrollo de habilidades básicas de crianza, disciplina y apoyo afectivo se reduce la presencia de conductas de riesgo. Así, los padres representan un factor clave en la prevención de conductas de riesgo. En este sentido, de acuerdo con Gallegos (2013) es tarea de padres y madres establecer con claridad las responsabilidades, derechos y límites a sus adolescentes, pues se reducirá el riesgo ante diversas circunstancias de su entorno como la violencia, las adicciones, la delincuencia, entre otras. Además de asegurar un clima relacional positivo, ya que ese ambiente será la base para construir las estrategias necesarias para afrontar las situaciones adversas presentes en la cotidianeidad (Viveros \& Vergara, 2014). Ambas tareas permitirán, no sólo mirar a la familia como agente de prevención de riesgo, sino también comprenderla, en palabras de Donas Burak (2001) como un factor protector de amplio espectro.

\section{Método}

La metodología se basa en la Investigación Acción Participativa (IAP) haciendo hincapié en una rigurosa búsqueda de conocimientos, un proceso abierto de vida y de trabajo, una vivencia hacia la transformación con objetivos sucesivos (Fals-Borda \& Rahman, 1989). De acuerdo con Ander-Egg (2003) la IAP supone tres grandes fases, diagnóstico, intervención y evaluación, que se constituyen por determinados pasos y características; y que no ocurren en una secuencia lineal, sino más bien circular. De manera particular, esta experiencia comprende tres fases: la primera fue un diagnóstico social, la segunda una intervención psicoeducativa y la tercera, una evaluación. Se realizó un diagnóstico social para determinar cuáles son las situaciones presentes y conductas predisponentes de los adolescentes que pudieran representar un riesgo para su salud. Lo anterior se llevó a cabo con la administración de un instrumento a los y las estudiantes de secundaria que evaluaba conductas cotidianas de los adolescentes, las cuales pudieran estar relacionadas con algún factor de riesgo.

Por otro lado, la intervención psicoeducativa se orientó hacia la identificación y prevención de los factores de riesgo a los que están expuestos los adolescentes, teniendo como actores principales a los padres y madres de familia, considerando tres principios en el quehacer psicoeducativo, considerados por autores como Bisquerra Alsina (1998)y
Santana Vega (2003): a) Principio de Prevención que concibe la intervención grupal como un proceso que prevé situaciones que pudieran obstaculizar el desarrollo integral de las personas y pretende impedir que un problema se presente o contrarrestar sus efectos. b) Principio de Desarrollo que considera cómo en la etapa de escolarización, el sujeto no sólo afronta cambios propios de su desarrollo evolutivo, sino que surge un nuevo contexto de relaciones y exigencias. Finalmente, c) Principio de Acción Social que se define como la posibilidad de que el sujeto haga un reconocimiento de variables contextuales y, de esta manera, pueda hacer uso de sus conocimientos desarrollados en la intervención.

La tercera fase de evaluación fue de corte cualitativo y se llevó a cabo mediante una evaluación de procesos y una evaluación final. La primera se centró en analizar la comprensión de las temáticas y promover que los participantes pudieran reconocer y ejemplificar lo visto en cada sesión, para poder prevenirlo en casa. La evaluación final se realizó para analizar la comprensión general de los factores de riesgo a los que están expuestos los adolescentes y contar con un espacio para compartir los aprendizajes construidos.

\section{Técnicas}

La IAP recurre a las técnicas tradicionales de la investigación social de una manera flexibilizada y con ciertas particularidades que resultan de la participación de la gente y los fines que se persiguen (Ander-Egg, 2003). Para la presente investigación se recurrió a diferentes técnicas, cada una de acuerdo a la fase en la que se encontraba el programa y a la población con la que estaba trabajando, siendo divididas de la siguiente manera:

En la primera fase se realizaron entrevistas estructuradas a dos profesoras, con el objetivo de conocer la perspectiva del personal docente acerca de los riesgos que perciben en el entorno de los adolescentes. Se contó con una guía que aborda temas como: la relación y comunicación entre compañeros, maestros y padres; los principales problemas que se observan en los alumnos y los cursos o talleres que se imparten a los jóvenes. Por otro lado, a los y las estudiantes se administró una encuesta centrada en las acciones que realizan los y las adolescentes en la actualidad e identificar a través de ellas las situaciones de riesgo a las que pueden estar expuestos.

El cuestionario administrado se denominó ¿Qué hacemos actualmente los jóvenes? que fue construido para esta investigación. Se integra de tres apartados. El primero se centra en acciones que suelen realizar los jóvenes en la adolescencia, lugares y personas que frecuentan y que los rodean; se conforma de 61 reactivos con opciones de sí o no, abordando las siguientes problemáticas: el Bullying, la violencia en la familia, la delincuencia, el alcoholismo, la drogadicción, el embarazo adolescente, las enfermedades de transmisión sexual y las autolesiones. El segundo apartado se centra en identificar los problemas que ellos perciben que se presentan en jóvenes de su edad, de manera general, en 
compañeros y compañeras de su escuela y en sus amigos y amigas cercanas; consta de tres reactivos donde se pide numerar las respuestas en orden de cotidianeidad con la que perciben cada situación. Finalmente, el tercer apartado indaga respecto a las problemáticas que cada adolescente enfrenta, si ha buscado ayuda para resolverlas y si su familia ha sido fuente de apoyo; se integra por tres reactivos abiertos.

De manera adicional, los y las estudiantes participaron en el diseño de un cartel con el objetivo de que lograran expresar a través de imágenes y algunas frases lo que saben respecto a las problemáticas que viven como adolescentes, lo que han observado de amigos cercanos o que ellos mismos viven. Para la administración de esta técnica se les pidió a los alumnos que, en equipos, plasmaran a través de un dibujo, la forma en la que ellos observan y perciben que se presentan las problemáticas, mencionadas anteriormente, en jóvenes de su edad; para ello se les repartió el material necesario (cartulinas, colores, plumones, lápices).

En la segunda fase, de Intervención psicoeducativa, se desarrolló un taller, el cual se entiende como una metodología de formación que permite abordar diferentes temáticas especialmente amplias; fomentando la participación, la cooperación, la reflexión y el diálogo como un medio de enriquecimiento y para la construcción del conocimiento (Escuelas promotoras de salud, 2014). Entre las técnicas utilizadas en el taller se encontraron las siguientes:

a) Técnica de Presentación Grupal: Tenía como objetivo que los participantes se conocieran y se creara un ambiente de confianza para poder desarrollar las actividades del taller. b) La Exposición Oral se llevó a cabo para que los padres puedan identificar las situaciones cotidianas en las que sus hijos e hijas se encuentran, con técnicas audiovisuales. c) El Debate se implementó con la finalidad de que los padres y madres de familia expusieran sus argumentos respecto a las causas de algunas problemáticas d) El Role Playing se realizó para representar las decisiones que tomarían y la forma en la que actuarían si ellos fueran adolescentes y sus "amigos" los incitaran a consumir alcohol.

En la última fase, para la evaluación de procesos se recurrió a la lluvia de ideas y un cuestionario breve. Para la evaluación final, las técnicas utilizadas fueron el grupo de discusión y el mural. El Grupo de discusión se utilizó para evaluar los conocimientos adquiridos a lo largo del taller y que las madres y padres pudieran expresar sus aprendizajes y el mural se utilizó para el cierre del taller con la finalidad de que los padres y madres plasmaran mensajes positivos en donde invitaran a los menores a tener una mejor comunicación entre sí.

\section{Participantes}

En el diagnóstico social participaron dos Profesoras de la escuela y 191 alumnos de segundo grado de una Es- cuela Secundaria ubicada en el Sur de la Ciudad de Mérida, de los cuales 98 (51.3\%) eran mujeres y 93 hombres $(48.7 \%)$ con edad promedio de 13.5 años. Para la intervención participaron 36 padres y madres de familia, 31 (86\%) fueron mujeres y cinco hombres (14\%).

\section{Escenario}

La investigación se desarrolló en una Escuela Secundaria de la Colonia Santa Rosa, ubicada en la zona sur del municipio de Mérida, Yucatán. Dicha zona es considerada con alto grado de marginación; en situaciones de pobreza y exclusión (Comisión Nacional para prevenir y erradicar la Violencia contra las Mujeres, 2009). Los bienes y servicios son de calidad deficiente; por lo que, los habitantes se trasladan al centro para cubrir sus necesidades (Cruz, 2009).

\section{Consideraciones éticas}

En primera instancia, se explicó a las autoridades de la Escuela Secundaria el objetivo principal de la investigación y la estrategia metodológica. Una vez obtenida su autorización se procedió a desarrollar el trabajo de investigación. Tanto a los alumnos como a los padres y las madres, primeramente, se les presentó el objetivo de a investigación, la importancia que tendría su participación; así como la pertinencia de reconocer la labor de los padres, madres, docentes y adolescentes para la prevención.

Posteriormente, se les invitó a participar de forma activa y comprometida; asegurándoles la confidencialidad de la información, el anonimato; así como la construcción de un espacio seguro para el diálogo y la atención más individualizada, en caso de requerirla. El trabajo con los alumnos, el personal docente y los padres y madres de familia se desarrolló siempre de manera respetuosa. Finalizado el trabajo, se socializaron, de forma clara y precisa, los resultados y límites del trabajo de investigación desarrollado.

\section{Resultados}

\section{Primera fase}

Los factores de riesgo principales, identificados en los y las estudiantes participantes, se encuentran el Bullying (51\%), la violencia familiar (48\%) y el alcoholismo $(47 \%)$, los cuales predisponen a los adolescentes a encontrarse en riesgo (ver Gráfica 1).

Factores identificados en el instrumento ¿Qué hacemos actualmente los jóvenes?

El bullying fue el factor de riesgo que los adolescentes reconocieron con mayor frecuencia. Es importante destacar que el $6 \%$ de los participantes señalan que las agresiones físicas son las conductas comunes que realizan los jóvenes 
Gráfica 1. Factores identificados en el instrumento ¿Qué hacemos actualmente nosotros los jóvenes?

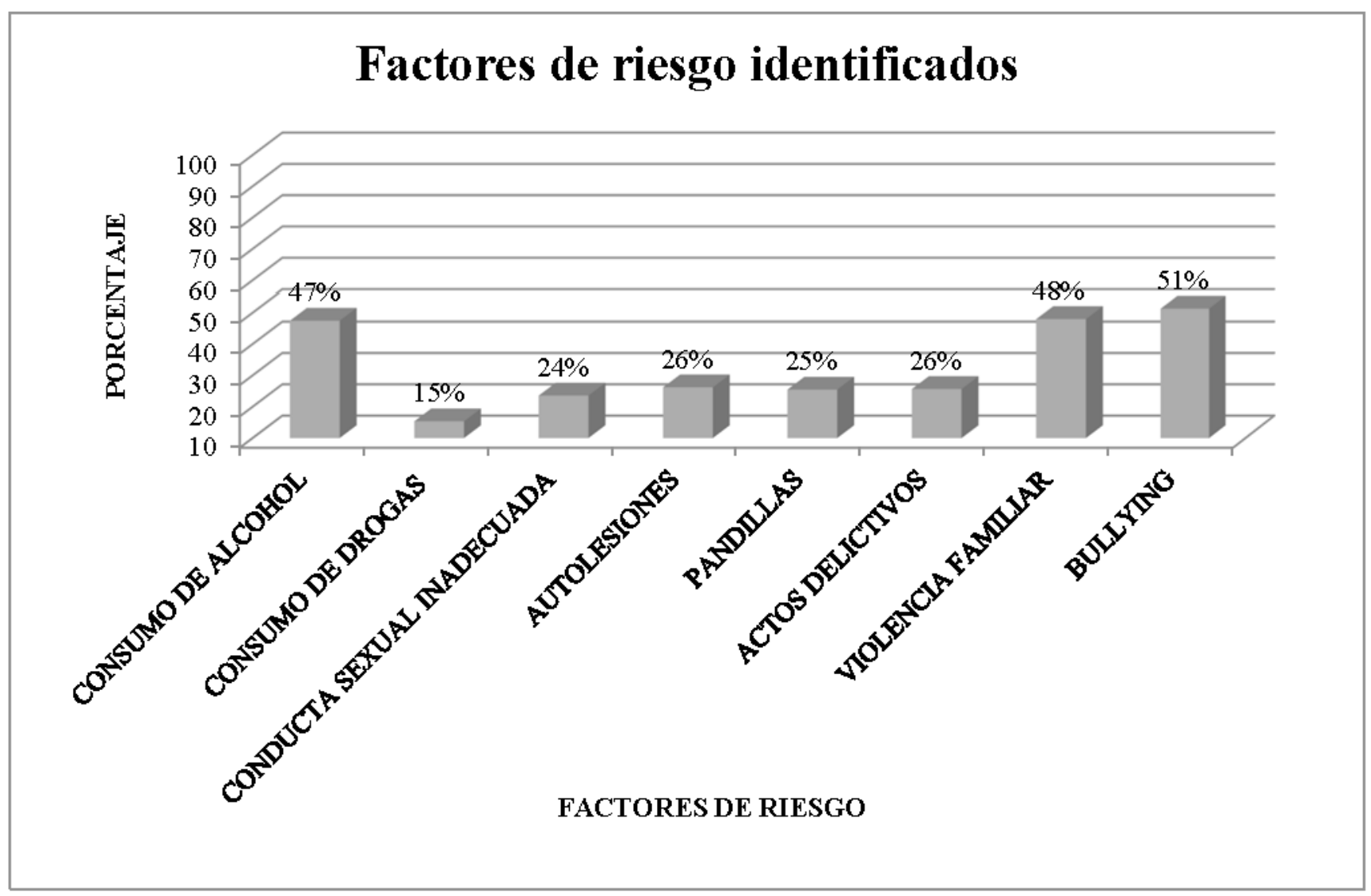

en la escuela, a través de golpes y/o lesiones físicas. El 10\% señala que suelen agredirse entre sí de manera verbal por medio de burlas de todo tipo, especialmente centradas en la apariencia física. El 3\% destaca que las burlas más que presentarse cara a cara, se realizan a través de internet. Adicionalmente, el $8 \%$ de los participantes identifica la exclusión de algunas personas respecto al grupo, como una conducta habitual en el entorno escolar. El 5\% señala que en algún momento se ha sentido ignorado por sus compañeros y el $8 \%$ indica que suele aplicarse la ley del hielo. Finalmente, el $4 \%$ identifica las amenazas de agresión como conductas presentes entre compañeros.

El segundo factor señalado con mayor presencia por los adolescentes fue la violencia familiar. El $9 \%$ de los participantes señala que cuando se portan mal reciben golpes por parte de sus padres; el $39 \%$ señala que suelen presentarse frecuentes peleas con sus padres; el $8 \%$ señaló que suelen llamarlos por apodos en su casa y el $5 \%$ indica que perciben un trato agresivo hacia ellos en casa. De manera adicional, se encontró que el $9 \%$ de los participantes señala que en su casa suelen resolverse los problemas con insultos y golpes y el $17 \%$ dice que sus padres tienden a pelear constantemente. El maltrato recibido constituye un tipo de violencia directa, mientras que el ser testigo de la violencia entre los padres constituye un tipo de violencia indirecta con importantes consecuencias para los menores (Bastarrachea, n.d.).

El tercer factor identificado fue el consumo de alcohol. El $47 \%$ de los adolescentes señaló que sus amigos suelen consumir alcohol, el $42 \%$ de los adolescentes dice que en las fiestas a las que normalmente asiste suele haber alcohol y el $6 \%$ indica que cuando en una fiesta ya no hay alcohol prefieren retirarse. El 6\% afirma consumir bebidas alcohólicas, mientras que el $5 \%$ indicó que ha llegado tarde a clases por haber bebido alcohol. En contraste a lo anterior, el $45 \%$ de los participantes señaló que controla su deseo de beber alcohol y el $30 \%$ dicen que los regañan sus padres si beben alcohol. Estos resultados evidencian cómo, de acuerdo con Escalante y Sánchez en Vázquez (2008) el alcohol es una de las principales sustancias consumidas por la población estudiantil y los amigos representan una gran influencia.

A través de los carteles se observó que los alumnos cuentan con gran cantidad de información acerca de los factores de riesgo, la cual les permite identificar el peligro en determinadas conductas. Por ejemplo, en los carteles de sexualidad destacan los comentarios acerca de la importancia de usar preservativo para tener relaciones sexuales, ya que protege contra Infecciones de Transmisión Sexual y embarazos no deseados. Por otro lado, en los carteles de delincuencia se resalta el que muchos jóvenes no respetan 
las normas y debido a ello, se involucran en problemas que pueden traer como consecuencia ir a prisión. Sobre los carteles de consumo de sustancias resaltan algunas consecuencias fisiológicas de éstas y señalan que muchos jóvenes no están informados de las mismas, especialmente en relación al consumo de alcohol, que es el más común. En relación con los carteles de autolesiones, comentan que esta situación se presenta en personas de su edad y que han sabido de algunos casos que son resultado de los problemas familiares. En relación a los carteles de pandillerismo, externan que existen muchas pandillas juveniles en su entorno y que muchas veces los jóvenes se dejan llevar por la popularidad o la pertenencia a un grupo, pero no miden las consecuencias y acaban realizando actos ilegales. En relación a los carteles de bullying, los alumnos señalan que éste está constantemente presente en el ámbito escolar, pues entre ellos mismos se ponen apodos o sobrenombres despectivos. También señalan que hay compañeros que se pelean a golpes, se insultan y se amenazan. Además de que no saben distinguir entre llevarse fuerte y la violencia con la cual lastiman a otros.

Finalmente, en los carteles de violencia familiar comentan que muchas veces la comunicación entre padres e hijos no es buena y que hay muchos padres y madres de familia que optan por el maltrato como medio de "educar" a los hijos. Comentan que en ocasiones algunos de ellos han pasado por esa situación. Situación que contribuye al riesgo de los adolescentes, ya que ellos señalan que cuando se sienten con confusión y dudas evitan preguntarles a sus padres y madres, por temor al maltrato. Por el contrario, recurren a amigos(as) o compañeros(as) quienes a su vez comparten sus mismos cuestionamientos y sus decisiones no se sustentan en información adecuada.

Con base en los anteriores resultados, se identificaron como principales problemáticas a trabajar en la intervención con padres y madres de familia, el bullying, la violencia familiar y el alcoholismo. La propuesta de intervención se presentó a las autoridades de la Escuela para su aprobación y posteriormente, se invitó a los padres y madres a participar.

\section{Segunda Fase}

El objetivo general fue brindar a los padres y madres de familia información acerca de los principales factores de riesgo a los cuales se encuentran expuestos sus hijos e hijas; así como herramientas básicas que promuevan la comunicación y la prevención en los menores. Los objetivos específicos fueron: A) Dar a conocer a los padres y madres participantes el trabajo de investigación en proceso. B) Explicar el bullying, la violencia familiar y el alcoholismo como factores de riesgo en los adolescentes, con la finalidad de que los padres y madres puedan identificarlos y realizar acciones para prevenirlo. C) Evaluar la comprensión, de los padres y madres, acerca de cada factor de riesgo; así como la identificación de acciones de prevención. Finalmente, D) Evaluar la importancia y utilidad del contenido del taller para los participantes tomando como base su rol y experiencia como educadores.

En la primera sesión se dio a conocer a los padres y madres participantes el trabajo de investigación en proceso, los resultados obtenidos durante el diagnóstico realizado con los adolescentes; así como el objetivo y las generalidades del taller a desarrollar. Al final de la sesión se realizó una actividad donde se formalizó el compromiso con el grupo y se discutió acerca de la importancia de conocer los factores de riesgo a los que se encuentran expuestos los adolescentes y poder tomar medidas de prevención. Finalizada esta sesión, el $61 \%$ de los padres y madres señalaron que aprendieron la importancia de que exista confianza y una buena comunicación con sus hijos e hijas, para que como familia logren identificar cuáles son los riesgos a los que están expuestos los adolescentes y de qué forma pudieran prevenirlo.

La segunda sesión se centró en el bullying, se explicó en qué consiste éste, como factor de riesgo y sus principales características. También se analizaron las principales consecuencias que puede tener el bullying para los adolescentes; así como la importancia de realizar acciones de prevención. En esta sesión, los participantes, propusieron acciones de prevención que pudieran realizar en casa. Entre ellas destacaron: platicar con sus hijos e hijas y darles información; así como estar pendientes de sus actitudes y acciones para detectar algún cambio que les sugiera que pueden estar pasando por una situación de violencia en la escuela; escucharlos, comprenderlos, mantener una buena comunicación como familia y poner en práctica algunos valores, entre otras. Al final de la sesión, el $90 \%$ de los padres y madres logran identificar al bullying como un problema que se presenta en el ámbito escolar y que consiste en agredir, ya sea física o verbalmente a una persona; así como que dichas acciones pueden ser realizadas por una persona o por un grupo de personas; también identifican que los involucrados son la víctima, el agresor y los observadores o espectadores.

La tercera sesión tenía como finalidad exponer la violencia familiar como factor de riesgo para los adolescentes y que los padres y madres lograran identificarlo para realizar acciones de prevención. Durante esta sesión se logró explicar a todos los participantes en qué consiste la violencia familiar como factor de riesgo para los adolescentes, así como las características. Del mismo modo, se analizaron 3 consecuencias que puede tener la violencia familiar en los adolescentes, así como la importancia de realizar acciones de prevención. Al finalizar la sesión, los participantes propusieron 5 acciones que pueden realizar como padres y madres de familia para la prevención de la violencia familiar. Algunas de ellas son las siguientes: Evitar regaños en público, supervisar y vigilar, evitar discusiones frente a los hijos, tener buena comunicación, identificar actitudes y expresiones de los adolescentes, evitar malas palabras en casa, evitar golpes, elevar el autoestima dándoles confianza y motivación; además de reconocer sus logros, corregir sus errores, establecer acuerdos y normas, cumplir reglas, evitar comparaciones entre hermanos, darles confianza y seguri- 
dad, apoyarlos en su educación, involucrarnos en las relaciones que establecen y transmitirles ideas correctas.

La cuarta sesión se centró en el alcoholismo, se logró que todos los participantes identificaran el alcoholismo como factor de riesgo en los adolescentes, así como las principales características y la forma en la que se presenta el consumo. Se analizaron, junto con los participantes, 3 consecuencias que puede tener el alcoholismo en los adolescentes, así como la importancia de realizar acciones de prevención. Se mencionaron algunos centros o instituciones que los padres y madres conocen para recibir ayuda ante el alcoholismo como: A.A (Alcohólicos Anónimos), Al-anon, Al-teen, Oceánica, y las pláticas brindadas en el seguro social (IMSS, ISSSTE) y otras instituciones como INJUVE y Centros de Adicciones. El hecho de que los padres y madres de familia cuenten con esta información puede ser un indicador positivo de su interés por informarse. Los padres y madres de familia señalan que entre las principales consecuencias que identifican que conlleva el alcoholismo se encuentran, la salud, el aprendizaje y rendimiento escolar y sus relaciones interpersonales.

\section{Tercera Fase}

Las madres y padres participantes resaltaron la importante de que se realicen actividades como este taller, ya que les ayuda a conocer y entender cómo es el ambiente de la escuela, cómo se desarrollan sus hijos e hijas y les permite mejorar sus relaciones. Sin embargo, señalaron que el tiempo destinado a las sesiones no fue suficiente, ya que consideran deben realizarse talleres de manera periódica para reforzar sus conocimientos. Adicionalmente, reconocen la necesidad de asesorías que les ayuden a identificar y solucionar los problemas que enfrentan sus hijos e hijas. Por otro lado, los padres y madres perciben que su asistencia a la escuela les da a sus hijos un sentimiento de bienestar y seguridad, ya que evidencian preocupación e interés por su educación.

Las madres y padres concuerdan en que, como cuidadores primarios, es fundamental que se involucren y participen en las actividades que se relacionan con la educación de sus hijos e hijas, ya que la responsabilidad de la educación recae en ellos, los profesores y los mismos adolescentes. Asimismo, externaron que al término del taller sentían estar seguros de la importancia que tiene acercarse más a sus hijos e hijas, guiarlos y protegerlos de aquellos riesgos ante los que se enfrentan. Expresan que las explicaciones les ayudaron para tener la información básica en cuanto a los factores de riesgos presentes en el entorno de los y las jóvenes, pero el uso constante de ejemplos les permitió comprender mejor e identificar los comportamientos en sus hijos e hijas.

Finalmente, en la técnica del mural, los padres y madres plasmaron mensajes positivos dirigidos a sus hijos e hijas y a todos los alumnos de la escuela secundaria. Estos expresaban sentimientos de confianza y comprensión ante los cambios por los que pasan los jóvenes durante la adolescencia; así como también mensajes que invitaban a los adolescentes a mantener una buena comunicación con sus familias. Finalizada la intervención, los padres y madres expresaron su inquietud por informarse respecto a otras temáticas actuales como el uso de la tecnología y las relaciones entre jóvenes.

Los padres y madres de familia recalcan la importancia de mantener la confianza y una buena comunicación con sus hijos e hijas, ya que esto es trascendente para lograr prevenir la influencia que los factores de riesgo pudieran tener en los adolescentes.

\section{Discusión}

Es importante destacar cómo la familia puede fungir como un factor protector, pero a la vez, puede ser un factor de riesgo (Blum \& Halcón, 2003; Zubarew \& Laval, 2003). En este caso, la presencia de la violencia familiar como uno de los factores de riesgo principales, evidencian la necesidad de generar procesos de investigación e intervención los cuales permitan comprender como señalan Welter y Scotegagna (2016) que la vulnerabilidad que permea la vida de estos adolescentes no se restringe apenas a un contexto personal o individual, no es un proceso lineal, pues la fragilidad proviene también de la familia, del medio social y de la escuela en que están insertos.

Considerando los factores de riesgo a los que los adolescentes están expuestos es imprescindible promover que los padres y madres de familia asuman un papel activo. Primero, reconociéndose como piezas clave en la prevención de conductas de riesgo. Segundo, involucrándose en procesos de capacitación en el desarrollo de habilidades básicas de crianza, disciplina y apoyo afectivo, los cuales, de acuerdo con Valenzuela y cols. (2013) reducen la presencia de conductas de riesgo.

De manera adicional, la familia y la institución escolar, en alianza con políticas públicas de salud, debe asumir el compromiso de monitorear los comportamientos relacionados a la salud de los adolescentes. Al mismo tiempo, se deben generar estrategias educativas que integren la labor de las secretarías de educación y de salud (Pena \& cols., 2016).

La estrategia metodológica que se siguió en este estudio resultó ser adecuada para el cumplimiento de los objetivos planteados. Sin embargo, hubiera sido importante desarrollar un proceso de intervención con los propios adolescentes.

\section{Futuras líneas de investigación y acción}

Se sugiere para futuras investigaciones realizar un diagnóstico lo más integral posible, es decir, tomar en cuenta a todas aquellas personas que intervienen en que se presente el acontecimiento estudiado, ya que de esa manera se enriquece la investigación y permite elaborar un trabajo de intervención de mejor calidad. Resultaría de gran importan- 
cia generar estrategias de intervención en las que se involucrara tanto a los y las adolescentes, profesores, padres y madres de familia en un mismo espacio, de tal manera que todos pueden recibir la misma información pero que cada quien pueda ejemplificar y plantear dudas desde su rol; ya que así se pudiera ampliar la comprensión de las temáticas al escucharse unos a otros y entre todos, proponer medidas que sean justas para todos y que los protejan ante los diversos riesgos a los que pueden estar expuestos. Los adultos significativos son esenciales debido a la influencia que tienen para el desarrollo de hábitos sanos para el adolescente, así como expresiones de afecto, relaciones interpersonales, resolución de conflictos y desarrollo del autocuidado. También es importante el efecto que tienen los estilos de crianza ya que pueden ser positivos o negativos para el desarrollo de conductas de riesgo (Valenzuela\& cols., 2013).

\section{Referencias}

Ander-Egg, E. (2003). Repensando la Investigación-AcciónParticipativa. Comentarios, críticas y sugerencias. Buenos Aires: Lumen.

Banco Mundial (2012). La violencia juvenil en México. Reporte de la situación, el marco legal y los programas gubernamentales. Recuperado de http://www.defensoria.ipn.mx/Documents/ Promotores/Estudio-Violencia-juvenil-en-Mexico-BM.pdf

Bastarrachea, G. (n.d.). La violencia familiar y sus secuelas en la adolescencia. Extraño iceberg de la violencia doméstica. Recuperado de http://www.codajic.org/sites/www.codajic.org/files/ El\%20iceberg\%20de\%20la\%20violencia\%20familar.pdf

Bisquerra Alzina R. (1998) Modelos de Orientación e Intervención Psicopedagógica. Barcelona: Editorial Praxis.

Blum, R.; Halcón, L. (2003). Adolescent heath in the Caribbean: Risk and Protective Factors. Am J Public Health,93, 456-60.

Comisión de Derechos Humanos del Estado de Yucatán (2010). Informe Especial sobre el Suicidio en Jóvenes del Estado de Yucatán. Yucatán: Comisión de Derechos Humanos del Estado de Yucatán.

Comisión Nacional para prevenir y erradicar la Violencia contra las Mujeres. (2009). Diagnóstico sobre la realidad social, económica y cultural de los entornos locales para el diseño de intervenciones en materia de prevención y erradicación de la violencia en la región sur: el caso de la zona metropolitana de Mérida, Yucatán. Recuperado de http://cedoc.inmujeres.gob.mx/lgamvlv/conavim/ merida.pdf

Consejo Nacional de Población (2014). Indicadores Sociodemográficos. Recuperado de http://www.conapo.gob.mx/ es/CONAPO/Indicadores_sociodemografico
Cruz, M. (2009). Diagnóstico sobre la realidad social, económica y cultural de los entornos locales para el diseño de intervenciones en materia de prevención y erradicación de la violencia en la región sur: el caso de la zona metropolitana de Mérida, Yucatán. México: Comisión Nacional Para Prevenir y Erradicar la Violencia contra las Mujeres. Recuperado de http://cedoc.inmujeres.gob.mx/ Igamvlv/conavim/merida.pdf

Cruz, R.; Ávila, M.; Velázquez, H.; Estrella, D. (2007). Evaluación de factores de riesgo de TCA en estudiantes de nutrición. Revista Mexicana de Trastornos Alimentarios, 4, 39-41. Recuperado: 12 nov. 2016. Disponível:http://www.scielo.org.mx/pdf/rmta/v4n1/ v4n1a5.pdf

Diario de Yucatán (2014). ¿Para qué un reglamento? Las normas que prohíben la instalación de establecimientos que venden bebidas embriagantes cerca de escuelas son letra muerta. Recuperado de http://yucatan.com.mx/merida/educacion-merida/para-que-unreglamento

Donas Burak, S. (2001). Marco epidemiológico conceptual de la salud integral y el desarrollo humano de los adolescentes. In: Donas Burak, S. (Org.), Adolescencia y juventud en América Latina (469487). Cartago: Libro Universitario Regional.

Encuesta Nacional de Juventud (2010). Resultados generales Yucatán. Disponivel: http://www.imjuventud.gob.mx/imgs/ uploads/3._ENJ_2010_-_Yucatan_VF_Mzo_16_MAC.pdf

Escuelas promotoras de salud (2014). Talleres. Guía Didáctica. Recuperado de http://www.epsuba.com.ar/cuerpo-docenteconvivencia-guia-didactica.php

Fals-Borda, O.; Rahman, M. A. (1989). La situación actual y las perspectivas de IAP en el mundo. In: Salazar, M. C. (Org.), La Investigación Acción participativa (pp. 205-223). Bogotá, Colombia: Cooperativa editorial Magisterio.

Gallegos, W. (2013). Agresión y violencia en la adolescencia: La importancia de la familia, Revista Avances en psicología, 21(1), 23-34. Recuperado: 25 oct. 2016. Disponível: http://www.unife. edu.pe/publicaciones/revistas/psicologia/2013/13_arias.pdf

Garduño, R.; Gómez K.; Peña, A. (2011). Suicidio en Adolescentes [Tesina]. Asociación Mexicana de Tanatología. Recuperado de http://www.tanatologia-amtac.com/descargas/tesinas/27\%20 Suicidio\%20en\%20adolescentes.pdf

Gobierno del Estado de Yucatán (2013). Ley para la Prevención, Combate y Erradicación de la Violencia en el Entorno Escolar del Estado de Yucatán.Disponível:http://www.poderjudicialyucatan. gob.mx/digestum/marcoLegal/05/2013/DIGESTUM05059.pdf

Infogen (2015). Trastornos alimenticios, anorexia, bulimia y por atracón. http://infogen.org.mx/trastornos-de-conducta-en-laalimentacion/ 
Instituto Nacional de Estadística y Geografía [INEGI] (2009). Estadística de suicidios de los Estados Unidos Mexicanos 2009. México: INEGI.

Instituto Nacional de Estadística y Geografía (INEGI) (2013). Estadísticas a propósito del día internacional de la juventud" datos de Yucatán. Disponível:http://www.inegi.org.mx/inegi/contenidos/ espanol/prensa/Contenidos/estadisticas/2013/juventud31.pdf

Instituto Nacional de Salud Pública (2013). Encuesta Nacional de Salud y Nutrición 2012. Resultados por entidad federativa. Yucatán. Disponível: http://ensanut.insp.mx/informes/YucatanOCT.pdf

Jiménez, R. (2005). La delincuencia juvenil: fenómeno de la sociedad actual, Papeles de Población, 11(43), 243-244.

Kiefer I.; Leitner, B.; Bauer, R.; Rieder A. (2000). Body weight: The male and female perception, SozPraventiv-med, 45 (6), 274-278.

Lila, M.; Buelga, S.; Musitu, G. (2006). Programa Lisis. Las relaciones entre padres e hijos en la adolescencia. Madrid: Ediciones Pirámide.

Lodewijks, H.;Doreleijers, T.; De Ruiter, C. (2008). SAVRY: Manual para la valoración estructurada de riesgo de violencia entre jóvenes. Generalitat de Catalunya. Departamento de justicia. Pp. $57-63$

Marín Cárdenas, A. D.; Sánchez Ramírez, G.; Maza Rodríguez,L. (2014).Prevalencia de obesidad y hábitos alimentarios desde el enfoque de género: el caso de Dzutóh, Yucatán, México, Revista Estudios Sociales, 22(44), 65-90.

Murueta, M.; Osorio, M. (2009). Psicología de la familia en Países Latinos del Siglo XXI. México: AMAPSI editorial. Recuperado de http://newpsi.bvs-psi.org.br/ebooks2010/pt/Acervo_files/ PsicologiaFamiliaLatinosXXI.pdf

Oliva-Peña, Y.; Ordóñez-Luna, M.; Santana-Carvajal, A.; MarínCárdenas, A. D.; Andueza Pech, G.; Gómez Castillo, I. A. (2016). Concordancia del IMC y la percepción de la imagen corporal en adolescentes de una localidad suburbana de Yucatán, Revista biomédica, 27 (2), 49-60.

Organización Mundial de la Salud (2013). Factores de riesgo. Recuperado de http://www.who.int/topics/risk_factors/es/

Pena, G. G.; Mendes, J. C. L.; Silveira, A. P.; Martins, T. C. R.; Vieira, R. G., Silva, N. S. S.; Silva, R. R. V. (2016). Comportamientos de riesgo para la salud de adolescentes de la red pública de enseñanza, Adolesc Saude, 13(1), 36-50.

Redondo Romero, A. (2013). Factores protectores a nivel familiar. Valencia: Universitat de Valencia.

Rocha, C.; Pacheco, M. (2008). Perfil del maltrato (bullying) entre estudiantes de secundaria en la ciudad de Mérida, Yucatán. Revista Mexicana de Investigación Educativa,13 (38), 829-834.

Rosabal García, E.; Romero Muñoz, N.; Gaquín Ramírez, K.; Hernández Mérida, R. A. (2015). Conductas de riesgo en los adolescentes. Rev Cubana Med Mil, 44(2), 218-229.

Sánchez Ramírez, E. C.; Dzib Aguilar, J. P. (2014). Autolesiones físicas en adolescentes yucatecos y su intervención. In: Frutos Cortés, M. (Org.) Violencia social y suicidio en el sureste de México (pp. 319329). Campeche: Secretaría de Salud del Estado de Campeche y Universidad Autónoma del Carmen.

Santana Vega, L. (2003) Orientación Educativa e Intervención Psicopedagógica. Madrid: Ediciones Pirámide.

Secretaría de Educación de Gobierno del Estado y Sindicato Nacional de Trabajadores de la Educación (2011). Guía para desarrollar Talleres de orientación con Padres y Madres de Familia. Yucatán: Secretaría de Educación de Gobierno del Estado y Sindicato Nacional de Trabajadores de la Educación.

Secretaría de Salud de Yucatán (2015). Programa de Violencia familiar. Yucatán: Secretaría de Salud de Yucatán.

Subsecretaría de Prevención y Participación Ciudadana (2011). Deserción Escolar y Conductas de Riesgo en Adolescentes. Recuperado de http://www.ssp.gob.mx/portalWebApp/ ShowBinary?nodeld=/BEA\%20Repository/1214166//archivo

Ulloa, R.; Hernández, C.; Paniagua K.; Figueroa, G. (2013). Frecuencia de autolesiones y características clínicas asociadas en adolescentes que acudieron a un hospital psiquiátrico infantil, Salud mental, 36 (5), 418-419.

Tarín, M.; Navarro J. (2006). Adolescentes en riesgo. Casos prácticos y estrategias de intervención socioeducativa. Madrid: Editorial CCS.

Valenzuela, M.; Ibarra, A.; Zubarew, T.; Loreto, M. (2013). Prevención de conductas de riesgo en el Adolescente: Rol de familia. Index Enferm, 22, 1-2.

Vázquez, D. (2008). Problemas más frecuentes en estudiantes de bachillerato. Tesis de Maestría, Universidad Autónoma de Yucatán. Recuperado de http://posgradofeuady.org.mx/wpcontent/uploads/2011/03/Tesis-Daniel-Vazquez.pdf

Viveros, E.; Vergara, C. (2014). Familia y dinámica familiar: cartilla dirigida a facilitadores para la aplicación de talleres con familias. Medellín: Fondo editorial FUNLAM. Recuperado de http://www.funlam.edu.co/uploads/fondoeditorial/preliminar/2014/ Familia-dinamica-familiar.pdf

Welter, P.; Scortegagna, S. A. (2016). Adolescentes que cometieron homicidio. ¿Cuáles son los factores de riesgo?, Adolesc Saude, 13(2), 7-15. 
Zubarew, T.; Laval, L. (2003). Guía para la supervisión de salud del adolescente. In: Zubarew, T.; Romero, M. I.; Poblete, F. (Org.), Adolescencia. Promoción, prevención y atención de salud. Ediciones Universidad Católica de Chile. Santiago de Chile, pp. 85-102.

Recebido em: 3 de janeiro de 2017 Aceito em: 01 de agosto de 2017 unrestricted use, distribution and reproduction in any medium, provided the original article is properly cited. 